\title{
A Joint-Reconstruction Approach for Single-Shot Edge Illumination X-Ray Phase-Contrast Tomography
}

\author{
Yujia Chen ${ }^{\mathrm{a}}$, Huifeng Guan ${ }^{\mathrm{a}}$, Charlotte K. Hagen ${ }^{\mathrm{b}}$, Alessandro Olivo ${ }^{\mathrm{b}}$, and Mark A. Anastasio ${ }^{\mathrm{a}}$ \\ aDepartment of Biomedical Engineering, Washington University in St. Louis, St. Louis, MO \\ 63130, USA \\ b Department of Medical Physics \& Biomedical Engineering, University College London, \\ London WC1E 6BT, UK
}

\begin{abstract}
Edge illumination X-ray phase-contrast tomography (EIXPCT) is an imaging technique that estimates the spatially variant X-ray refractive index and absorption distribution within an object while seeking to circumvent the limitations of previous benchtop implementations of X-ray phase-contrast tomography. As with gratingor analyzer-based methods, conventional image reconstruction methods for EIXPCT require that two or more images be acquired at each tomographic view angle. This requirement leads to increased data acquisition times, hindering in vivo applications. To circumvent these limitations, a joint reconstruction (JR) approach is proposed that concurrently produces estimates of the refractive index and absorption distributions from a tomographic data set containing only a single image per tomographic view angle. The JR reconstruction method solves a nonlinear optimization problem by use of a novel iterative gradient-based algorithm. The JR method is demonstrated in both computer-simulated and experimental EIXPCT studies.
\end{abstract}

Keywords: Joint reconstruction, phase-contrast imaging, single-shot imaging

\section{INTRODUCTION}

Edge illumination X-ray phase-contrast tomography (EIXPCT), also called coded aperture phase-contrast tomography, is a phase-contrast tomography (PCT) technique that is readily used in a laboratory setting. ${ }^{1} \mathrm{PCT}$ estimates the spatially variant distribution of the complex-valued X-ray refractive index distribution $n=1-\delta+i \beta$, where $\delta$ and $\beta$ denote the refractive index and absorption, respectively. Compared with other PCT technologies that were being developed for use with synchrotrons, ${ }^{2,3}$ EIXPCT is compatible with standard commercial laboratory-based X-ray equipment. ${ }^{1}$ The conventional two-step reconstruction algorithm for the EIXPCT system requires that two or more images are acquired at each tomographic view angle for the first phase-retrieval step. This requirement prolongs the data-acquisition time and increases the radiation dosage. Single-shot methods that can remove the need to acquire multiple images per view angle are highly desirable in EIXPCT because they can lead to reduced imaging times and radiation doses, thus facilitating in vivo applications. Previously proposed single-shot EIXPCT methods require restrictive assumptions. For example, scanning geometry assumptions require the incident X-ray wave to be parallel beam. ${ }^{4}$ Object uniformity assumptions require the objects to be homogeneous and the ratio between the absorption and refractive index to be constant for the whole object. ${ }^{5}$ Another single-shot method requires use of an energy-sensitive detector. ${ }^{6}$

To circumvent these requirements, a joint reconstruction (JR) approach is proposed that concurrently produces estimates of the refractive index and absorption distributions from a tomographic data set containing only a single image per tomographic view angle. The need for multiple measurements as well as a phase retrieval step at each view angle can be removed by directly exploiting the full tomographic data. This algorithm does not require any assumptions or restrictions concerning the object's material uniformity, the scanning geometry, or the detector type. To accomplish this, the JR method solves a non-linear optimization problem by use of a novel iterative gradient-based algorithm.

Further author information: (Send correspondence to Mark A. Anastasio)

Mark A. Anastasio: E-mail: anastasio@seas.wustl.edu, Telephone: 13149353637

Medical Imaging 2017: Physics of Medical Imaging, edited by Thomas G. Flohr,

Joseph Y. Lo, Taly Gilat Schmidt, Proc. of SPIE Vol. 10132, 1013217 · @ 2017 SPIE

CCC code: $1605-7422 / 17 / \$ 18 \cdot$ doi: $10.1117 / 12.2255545$

Proc. of SPIE Vol. $101321013217-1$ 


\section{IMAGING MODEL}

The basic setup for an EIXPCT system is described as follows. ${ }^{7}$ Two masks, denoted by $A_{1}$ (sample mask) and $A_{2}$ (detector mask), are employed to provide an edge-illumination setup that permits measurement of phasecontrast signals. The sample is placed immediately after sampling mask $A_{1}$. When the relative positions of the two parallel masks are changed, different parts of the incident X-ray beam fall onto the sensitive area of the detector, encoding differential phase information in the X-ray wave intensity. If we denote the distance from the source to $A_{1}$ as $z_{s o}$ and the distance between $A_{1}$ and $A_{2}$ as $z_{o d}$, then $A_{1}$ can be translated to select the operating regime of the system. The relative displacement of the two masks after the translation is denoted by $\Delta \xi$.

Let $\beta(\mathbf{r})$ and $\delta(\mathbf{r})$ represent the absorption and refractive index distributions, respectively. The stationary reference coordinate system is described by $\boldsymbol{r}=(x, y)$. In a tomographic scan, the origin of the reference system is the rotation center. A rotated coordinate system $\left(x_{r}, y_{r}\right)$ is related to the reference system by $x_{r}=x \cos \theta+y \sin \theta$, $y_{r}=y \cos \theta+x \sin \theta$, where the tomographic view angle $\theta$ is measured from the positive $\mathrm{x}$-axis.

For an incident parallel beam or an incident spherical wave in the paraxial limit, the beam propagates in the direction of the positive $x_{r}$-axis. Let $\mathcal{H}$ denote the two-dimensional (2D) Radon transform operator:

$$
\mathcal{H}\left(\theta, y_{r} ; \beta\right)=\int_{L\left(y_{r}, \theta\right)} \beta(\boldsymbol{r}) d x_{r}
$$

where $L\left(y_{r}, \theta\right)$ denotes a line that is parallel to the $x_{r}$-axis and goes through $\left(0, y_{r}\right)$. Let $\mathcal{D}$ denote the first-order derivative with respect to the detector coordinate $y_{r}$ of the 2D Radon transform:

$$
\mathcal{D}\left(\theta, y_{r} ; \delta\right)=\frac{\partial}{\partial y_{r}} \mathcal{H}\left(\theta, y_{r} ; \delta\right)=\frac{\partial}{\partial y_{r}} \int_{L\left(y_{r}, \theta\right)} \delta(\boldsymbol{r}) d x_{r} .
$$

When imaging soft tissue, the forward model for EIXPCT can be expressed as ${ }^{7}$

$$
I_{\Delta \xi}\left(\theta, y_{r} ; \beta, \delta\right)=\exp \left(-\frac{2 \pi}{\lambda} \mathcal{H}\left(\theta, y_{r} ; \beta\right)\right)\left[I_{T C}(\Delta \xi)-\frac{z_{o d}}{M} \mathcal{D}\left(\theta, y_{r} ; \delta\right) I_{T C}^{\prime}(\Delta \xi)\right]
$$

where $I_{\Delta \xi}$ is the measured intensity corresponding to mask displacement $\Delta \xi$ normalized by the incident X-ray wave intensity, $I_{T C}^{\prime}(\Delta \xi)$ denotes the 1-st order derivative of $I_{T C}(\Delta \xi), \lambda$ is the wavelength of the incident beam, and $M=\left(z_{s o}+z_{o d}\right) / z_{s o}$ is a magnification factor. The function $I_{T C}(\Delta \xi)$ is referred to as the illumination curve, and is acquired by a separate air-scan calibration procedure. ${ }^{7}$

Define

$$
A_{\Delta \xi} \equiv I_{T C}(\Delta \xi), B_{\Delta \xi} \equiv \frac{z_{o d}}{M} I_{T C}^{\prime}(\Delta \xi)
$$

and note that $A_{\Delta \xi}$ and $B_{\Delta \xi}$ are constants that can be determined after the calibration procedure. The optimal choice of $\Delta \xi$ has been discussed by Munro et. al. ${ }^{7}$ For single-shot imaging, no translation of the mask is required and $\Delta \xi=\eta_{1} P / 2 M .{ }^{7}$ In terms of these quantities, the forward model can be re-expressed as

$$
I_{\Delta \xi}\left(\theta, y_{r} ; \beta, \delta\right)=\exp \left(-k \mathcal{H}\left(\theta, y_{r} ; \beta\right)\right)\left[A_{\Delta \xi}-B_{\Delta \xi} \mathcal{D}\left(\theta, y_{r} ; \delta\right)\right],
$$

where the wave number $k=2 \pi / \lambda$. The goal of joint image reconstruction is to determine estimates of $\delta(\mathbf{r})$ and $\beta(\mathbf{r})$ by inverting Eq. (5) from the single-shot measurement data $I_{\Delta \xi}\left(\theta, y_{r} ; \beta, \delta\right)$ acquired at view angles $\theta \in[0,2 \pi)$.

\section{IMAGE RECONSTRUCTION}

A discrete imaging model needs to be established so that the inverse problem can be solved numerically. Let the vectors

$$
\boldsymbol{\beta}=\left[\beta_{1,1}, \beta_{1,2}, \ldots, \beta_{1, N_{x}}, \beta_{2,1}, \ldots, \beta_{N_{x}, N_{y}}\right]^{T} \in \mathbb{R}^{N}
$$


and

$$
\boldsymbol{\delta}=\left[\delta_{1,1}, \delta_{1,2}, \ldots, \delta_{1, N_{x}}, \beta_{2,1}, \ldots, \delta_{N_{x}, N_{y}}\right]^{T} \in \mathbb{R}^{N}
$$

represent the values of the absorption and refractive index sampled at the $N=N_{x} N_{y}$ vertices $\boldsymbol{r}_{i, j}=\left(x_{i}, y_{j}\right)$ $\left(i=1,2, \ldots, N_{x}\right.$ and $\left.j=1,2, \ldots, N_{y}\right)$ of a Cartesian grid. Let

$$
\begin{aligned}
\boldsymbol{I}(\boldsymbol{\beta}, \boldsymbol{\delta})= & {\left[\left(\theta_{1}, y_{r 1} ; \boldsymbol{\beta}, \boldsymbol{\delta}\right), I\left(\theta_{1}, y_{r 2} ; \boldsymbol{\beta}, \boldsymbol{\delta}\right), \ldots, I\left(\theta_{1}, y_{r Q} ; \boldsymbol{\beta}, \boldsymbol{\delta}\right),\right.} \\
& I\left(\theta_{2}, y_{r 1} ; \boldsymbol{\beta}, \boldsymbol{\delta}\right), I\left(\theta_{2}, y_{r 2} ; \boldsymbol{\beta}, \boldsymbol{\delta}\right), \ldots, I\left(\theta_{2}, y_{r Q} ; \boldsymbol{\beta}, \boldsymbol{\delta}\right), \ldots \\
& \left.I\left(\theta_{P}, y_{r 1} ; \boldsymbol{\beta}, \boldsymbol{\delta}\right), I\left(\theta_{P}, y_{r 2} ; \boldsymbol{\beta}, \boldsymbol{\delta}\right), \ldots, I\left(\theta_{P}, y_{r Q} ; \boldsymbol{\beta}, \boldsymbol{\delta}\right)\right]^{T} \in \mathbb{R}^{P Q}
\end{aligned}
$$

denote the stacked measured intensity data, where $\theta_{p}(p=0,1, \ldots, P)$ denote $P$ tomographic view angles, and $y_{r q}(q=0,1, \ldots, Q)$ denote the $\mathrm{Q}$ detector positions in the rotated coordinate system. Let $\boldsymbol{H} \in \mathbb{R}^{N} \rightarrow \mathbb{R}^{P Q}$ and $\boldsymbol{D} \in \mathbb{R}^{N} \rightarrow \mathbb{R}^{P Q}$ denote the discretized $2 \mathrm{D}$ Radon transformation operator and its first-order derivative, respectively. Then,

$$
[\boldsymbol{I}(\boldsymbol{\beta}, \boldsymbol{\delta})]_{i}=\exp \left(-\frac{2 \pi}{\lambda}[\boldsymbol{H} \boldsymbol{\beta}]_{i}\right)\left[A_{\Delta \xi}-B_{\Delta \xi}[\boldsymbol{D} \boldsymbol{\delta}]_{i}\right],
$$

where $i=1,2, \ldots, P Q$ denotes the index of the measured intensity data point.

Joint image reconstruction can be formulated as the following non-linear optimization problem:

$$
(\tilde{\boldsymbol{\beta}}, \tilde{\boldsymbol{\delta}})=\underset{\tilde{\boldsymbol{\beta}}, \tilde{\boldsymbol{\delta}}}{\arg \min } f(\tilde{\boldsymbol{\beta}}, \tilde{\boldsymbol{\delta}}),
$$

where $\tilde{\boldsymbol{\beta}}$ and $\tilde{\boldsymbol{\delta}}$ denote estimates of $\boldsymbol{\beta}$ and $\boldsymbol{\delta}$. In this study, objects are reconstructed by minimizing the square error between the estimated intensity and the experimentally measured intensity. Specifically, the objective function $f(\boldsymbol{\beta}, \boldsymbol{\delta})$ is assumed to be of the form

$$
f(\boldsymbol{\beta}, \boldsymbol{\delta})=\left\|\boldsymbol{I}_{m}-\boldsymbol{I}(\boldsymbol{\beta}, \boldsymbol{\delta})\right\|^{2}+R(\boldsymbol{\beta}, \boldsymbol{\delta}) .
$$

Here, $\boldsymbol{I}_{m}$ denotes the measured data and $\boldsymbol{I}(\boldsymbol{\beta}, \boldsymbol{\delta})$ denotes the simulated measurement data obtained by use of Eq. (5) for a given choice of $\boldsymbol{\beta}$ and $\boldsymbol{\delta}$. The penalty term $R(\boldsymbol{\beta}, \boldsymbol{\delta})$ can be designed to appropriately regularize the inverse problem, which is generally ill-posed. The studies below employed a differentiable total variation (TV) penalty. ${ }^{8}$

A batch gradient algorithm was developed to solve Eq. (10), with the pseudocode provided in Alg. 1. A simple stopping rule based on the value of the objective function was employed. The derivatives of $R(\tilde{\boldsymbol{\beta}}, \tilde{\boldsymbol{\delta}})$ for the case of a differentiable TV penalty have been proposed elsewhere. ${ }^{8}$

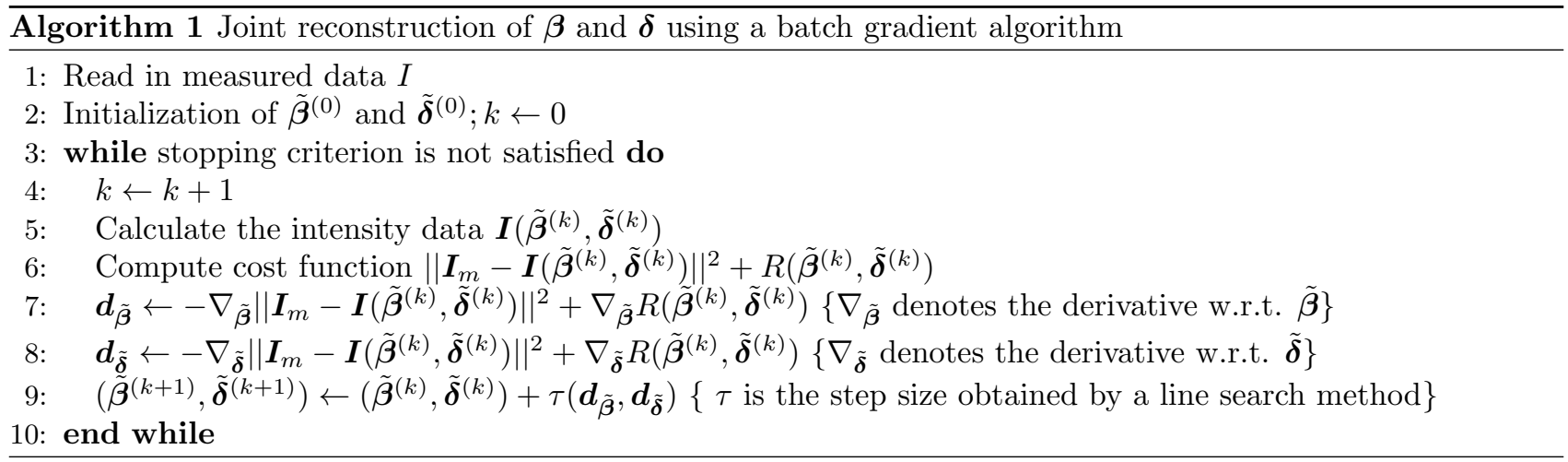




\section{SIMULATION STUDIES}

Computer-simulation studies were employed to demonstrate and validate the proposed image reconstruction method. In the computer-simulation study, a $256 \times 256$ pixel artificial beetle phantom adapted from a real beetle object $^{1}$ was employed to represent spatially variant distributions of the absorption and refractive index. The magnitudes of the absorption and refractive index values were on the orders of $10^{-10}$ and $10^{-7}$, respectively. The ratio of the absorption and refractive index was pixel dependent and not a constant over the whole image. Each element in the phantom represented a physical size of $10 \mu \mathrm{m} \times 10 \mu \mathrm{m}$. A monochromatic X-ray parallel beam of $10^{-10} \mathrm{~m}$ wavelength (i.e., $12.4 \mathrm{keV}$ ) was assumed to radiate the object. The two masks were positioned so that $z_{s o}=1.6 \mathrm{~m}$ and $z_{o d}=0.4 \mathrm{~m}$. By use of Eq. (3), measurement data corresponding to 720 tomographic views that were evenly distributed over 360 degrees were computed. The detector was assumed to contain 400 pixels $10 \mu \mathrm{m}$ wide. In these preliminary studies, no stochastic noise was added to the measurement data. The illumination curve was obtained from previous experimental data. ${ }^{7}$ For the optimal mask displacement $\Delta \xi=8 \mu m$, the value of $I_{T C}$ and the corresponding gradient $I_{T C}^{\prime}$ were determined from the illumination curve as $I_{T C}=0.5$ a.u. (arbitrary units), and $I_{T C}^{\prime}=-4 \times 10^{4} \mathrm{~m}^{-1}$. Algorithm 1 was employed to reconstruct estimates of $\delta(\mathbf{r})$ and $\beta(\mathbf{r})$. The algorithm was terminated when the value of the cost function fell below 0.008 . No penalty was employed in the reconstruction algorithm because noise was absent.

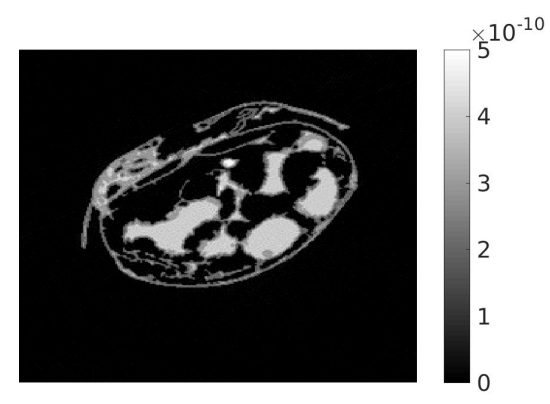

(a)

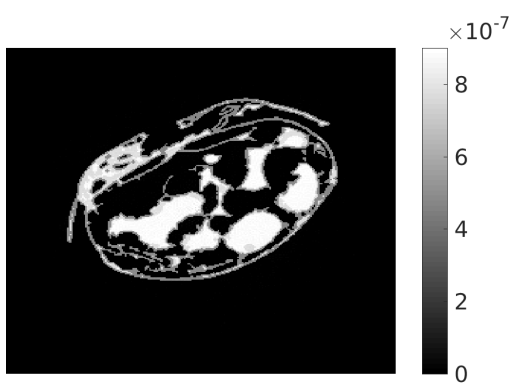

(d)

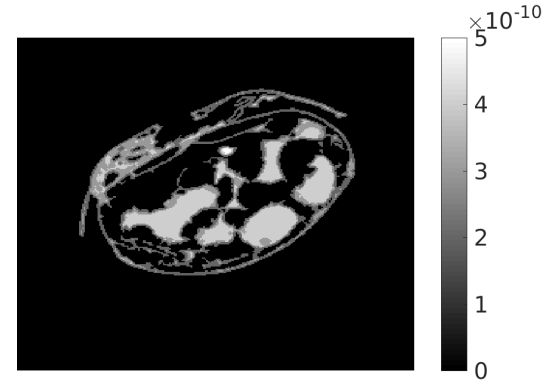

(b)

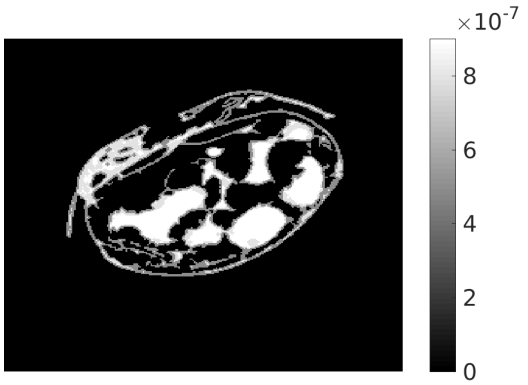

(e)

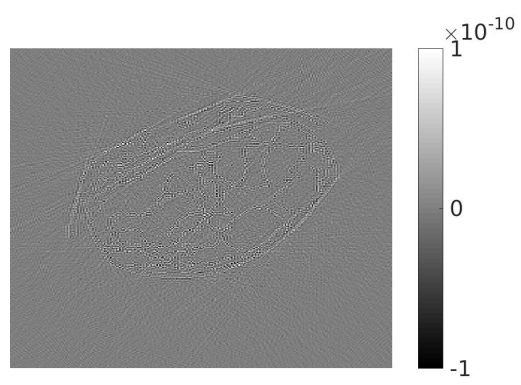

(c)

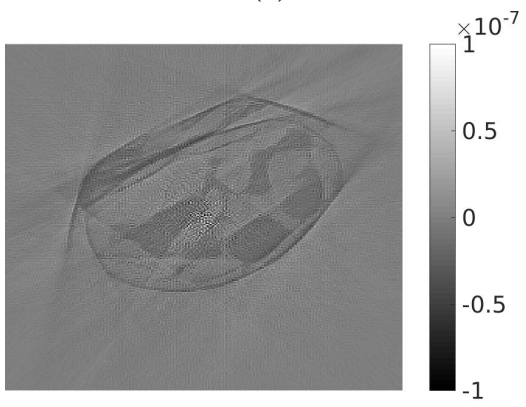

(f)

Figure 1: Reconstructed images from noise-free simulated measurement data. (a) Reconstructed absorption distribution; (b) true absorption distribution; (c) the difference between (a) and (b); (e) Reconstructed refractive index distribution; (f) true refractive index distribution; (g) the difference between (e) and (f);

Figure 1 shows reconstructed images from computer-simulated data. For the absorption, the mean square error (MSE) of the reconstructed distribution is $1.9 \times 10^{-22}$. For the refractive index, the corresponding MSE is $9.4 \times 10^{-17}$. These values are small compared with the actual intensity of the absorption and refractive index distribution. These results confirm the feasibility and accuracy of joint reconstruction under idealized conditions.

\section{EXPERIMENTAL STUDIES}

An experimental data set of a chicken bone sample produced by use of a benchtop EIXPCT system was also employed. ${ }^{4}$ The mean energy employed in the imaging studies was $17 \mathrm{keV}$. Tomographic data were acquired over a $360^{\circ}$ range, with an angular spacing of $0.5^{\circ}$. The imaging process involved eight dithering steps ${ }^{4}$ to increase 

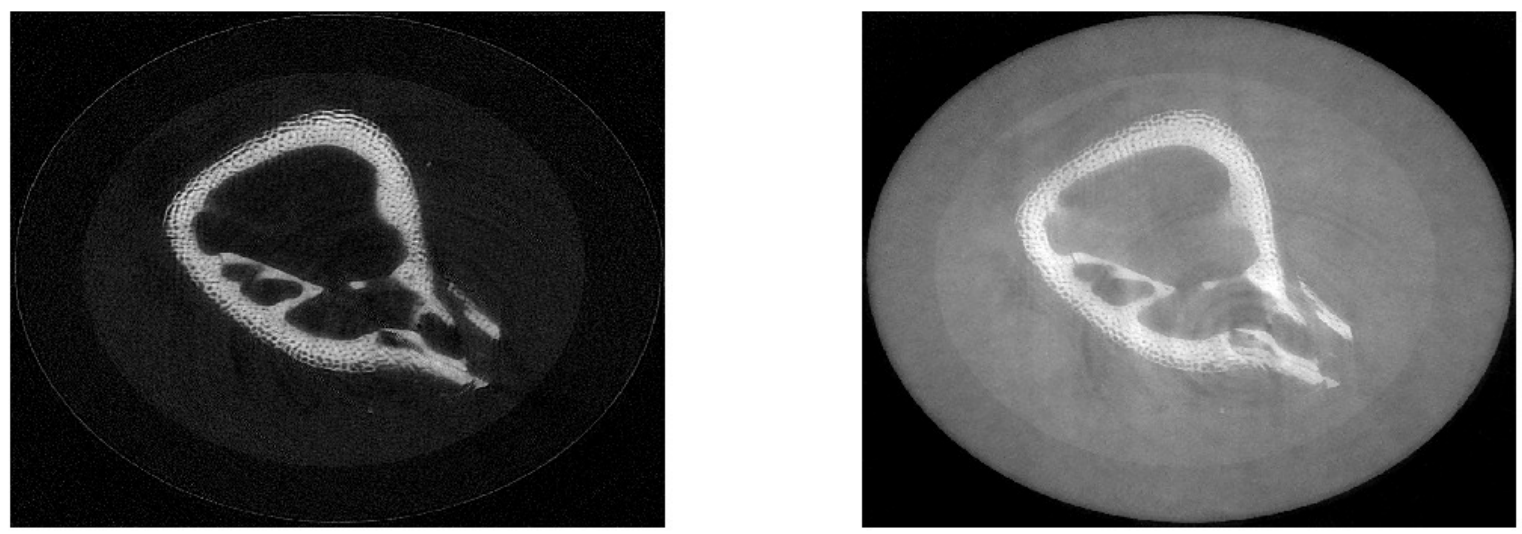

(a)

(b)

Figure 2: (a) Reconstructed absorption image for experimental chicken bone data. (b) Reconstructed refractive index image for experimental chicken bone data.

data resolution at the sub-pixel scale. The results produced from experimental data are shown in Fig. 2, where detailed features in the images are visible.

Note that in this specific case dithering was used to increase the spatial resolution in the final image to sub-pixel levels. Consequently, the same tomographic view angle was shot eight times, but the multiple shotting does not undermine the merit of the fast acquisition. The above reconstruction results still suggest that if a resolution equal to the pixel size can be considered sufficient, or the experimental setup can be further improved so that dithering is no longer needed for a super-resolution image, the joint reconstruction of absorption $\boldsymbol{\beta}$ and refractive index $\boldsymbol{\delta}$ from single-shot measurement data is feasible in a practical scenario.

\section{CONCLUSIONS}

A non-linear, gradient-based, joint image reconstruction algorithm for single-shot EIXPCT has been developed and investigated. This method is more general and practical because it does not require restrictive assumptions about the scanning geometry ${ }^{4}$ or object, ${ }^{5}$ or require use of an energy-sensitive detector. ${ }^{6}$ The method was validated by use of both computer-simulated and experimental EIXPCT data.

\section{ACKNOWLEDGMENTS}

This work was supported in part by NIH award EB02060401 and NSF award CBET1263988. C.K.H and A.O. were supported by EPSRC grants EP/L001381/1 and EP/I021884/1

\section{REFERENCES}

[1] Hagen, C. K., Diemoz, P. C., Endrizzi, M., Rigon, L., Dreoosi, D., Arfelli, F., Lopez, F. C. M., Longo, R., and Olivo, A., "Quantitative edge illumination x-ray phase contrast tomography," Proc. SPIE 9212, 921205-921205-11 (2014).

[2] Momose, A., Takeda, T., Itai, Y., and Hirano, K., "Phase-contrast X-ray computed tomography for observing biological soft tissues," Nature Medicine 2(4), 473-475 (1996).

[3] Snigirev, A., Snigireva, I., Kohn, V., Kuznetsov, S., and Schelokov, I., "On the possibilities of X-ray phase contrast microimaging by coherent high-energy synchrotron radiation," Review of Scientific Instruments $\mathbf{6 6}(12)$, 5486-5492 (1995).

[4] Hagen, C. K., Endrizzi, M., Diemoz, P. C., and Olivo, A., "Reverse projection retrieval in edge illumination X-ray phase contrast computed tomography," Journal of Physics D: Applied Physics 49(25), 255501 (2016). 
[5] Diemoz, P. C., Vittoria, F. A., Hagen, C. K., Endrizzi, M., Coan, P., Brun, E., Wagner, U. H., Rau, C., Robinson, I. K., Bravin, A., and Olivo, A., "Single-image phase retrieval using an edge illumination X-ray phase-contrast imaging setup," Journal of Synchrotron Radiation 22(4), 1072-1077 (2015).

[6] Das, M. and Liang, Z., "Spectral X-ray phase contrast imaging for single-shot retrieval of absorption, phase, and differential-phase imagery," Optics Letters 39(21), 6343-6346 (2014).

[7] Munro, P. R., Hagen, C. K., Szafraniec, M. B., and Olivo, A., "A simplified approach to quantitative coded aperture x-ray phase imaging," Optics Express 21(9), 11187-11201 (2013).

[8] Sidky, E. Y., Kao, C.-M., and Pan, X., "Accurate image reconstruction from few-views and limited-angle data in divergent-beam CT," Journal of X-ray Science and Technology 14(2), 119-139 (2006). 